\title{
Estresse ocupacional e comportamentos de saúde de enfermeiros de um hospital terciário de Fortaleza - Ceará
}

\author{
Occupational stress and health behaviors of nurses in a tertiary hospital in Fortaleza - Ceará \\ Estrés ocupacional y comportamientos de salud de enfermeras en un hospital terciario de Fortaleza - Ceará
}

\begin{abstract}
RESUMO
Objetivo: Identificar os fatores de estresse ocupacional e os comportamentos de saúde de enfermeiros de um hospital terciário de Fortaleza-CE. Método: Trata-se de um estudo transversal, desenvolvido em uma unidade hospitalar de atenção terciária de Fortaleza, no período de outubro de 2018 a março de 2019. Resultado: A amostra constituiu-se de 134 enfermeiros que foram recrutados por meio de amostragem por conveniência. Utilizou-se um questionário estruturado, pré-codificado, com 12 questões fechadas de múltipla escolha. Verificou-se que $26,1 \%$ (n=35) dos profissionais fazem uso de substâncias psicoativas e $61,9 \%(n=83)$ apresentaram de 1 a 5 sinais/sintomas de estresse. Conclusão: Destarte, este estudo será útil para que os gestores reflitam sobre os fatores que influenciam a qualidade de vida dos enfermeiros, contribuindo para implementação de ações que melhorem as condições de trabalho e favoreçam a proteção do trabalhador.
\end{abstract}

DESCRITORES: Profissionais de Enfermagem; Estresse Ocupacional; Esgotamento Profissional.

\section{ABSTRACT}

Objective: Identify occupational stress factors and health behaviors of nurses in a tertiary hospital in Fortaleza-CE. Method: This is a cross-sectional, developed in a tertiary care hospital in Fortaleza, from October 2018 to March 2019. Result: The sample consisted of 134 nurses who were recruited through convenience sampling. A pre-coded structured questionnaire was used, with 12 closed multiple questions. It was found that $26.1 \%(n=35)$ of the professionals use psychoactive substances and $61.9 \%$ $(n=83)$ had 1 to 5 signs/symptoms of stress. Conclusion: Thus, this study will be useful for managers to reflect on the factors that influence the quality of life of nurses, contributing to the implementation of actions that improve working conditions and favor worker protection.

[DESCRIPTORS: Nurse Practitioners; Occupational Stress; Burnout.

\section{RESUMEN}

Objetivo: Identificar factores de estrés ocupacional y comportamientos de salud de enfermeras de un hospital terciario de Fortaleza-CE. Método: Se trata de un estudio transversal realizado en un hospital terciario de Fortaleza, de octubre de 2018 a marzo de 2019. Resultado: La muestra estuvo conformada por 134 enfermeras que fueron reclutadas mediante muestreo por conveniencia. Se utilizó un cuestionario estructurado y precodificado con 12 preguntas cerradas de opción múltiple. Se encontró que el $26,1 \%(n=35)$ de los profesionales consumen sustancias psicoactivas y el $61,9 \%(n=83)$ presenta de 1 a 5 signos / síntomas de estrés. Conclusión: Así, este estudio será de utilidad para que los gerentes reflexionen sobre los factores que influyen en la calidad de vida de las enfermeras, contribuyendo a la implementación de acciones que mejoren las condiciones laborales y favorezcan la protección de los trabajadores.

DESCRIPTORES: Profesionales de Enfermería; Estrés laboral; Burnout profesional.

RECEBIDO EM: 09/09/2020 APROVADO EM: 10/09/2020

Francisco Ariclene Oliveira

Enfermeiro. Universidade Federal do Ceará.

ORCID: 0000-0002-0290-4797

Dayanna Cynthia Moura Melo

Enfermeira. Universidade Estadual do Ceará.

ORCID: 0000-0001-8270-3786 


\title{
artigo
}

Oliveira, F.A.; Melo, D.C.M.; Santos, A.P.L.M.; Menezes, C.P.S.R.; Sales, T.B.; Freitas, M.M.L; Afonso, L.R.;

Estresse ocupacional e comportamentos de saúde de enfermeiros de um hospital terciário de Fortaleza - Ceará

\author{
Ana Paula Lima Menezes dos Santos \\ Enfermeira. Universidade de Fortaleza. \\ ORCID: 0000-0002-4201-3139
}

\section{Cláudia Patrícia Da Silva Ribeiro Menezes}

Enfermeira. Faculdade de Quixeramobim.

ORCID: 0000-0002-0559-9302

\section{Tamara Braga Sales}

Enfermeira. Universidade Federal do Ceará.

ORCID: 0000-0003-1628-862X

\section{Maíra Maria Leite de Freitas}

Acadêmica do curso de Enfermagem da Universidade Federal do Ceará.

ORCID: 0000-0003-1150-8152

\section{Lucélia Rodrigues Afonso}

Enfermeira. Universidade Estadual do Ceará.

ORCID: 0000-0002-8370-4008

\section{INTRODUÇÃO}

0 processo de trabalho em saúde é complexo, dinâmico, contínuo e engloba mudanças socioeconômicas, políticas e tecnológicas. Com isso, as transformações abruptas nas atividades de trabalho afloram o estresse ocupacional e, consequentemente, leva ao adoecimento do profissional de saúde. Para tanto, o estresse ocupacional é advindo de um ambiente laboral deletério, interligado aos fatores organizacionais, às condições de trabalho e à qualidade das relações interpessoais no trabalho. Em consonância, o estresse ocupacional é decorrente das necessidades e/ou situações do trabalho onde o profissional está inserido, portanto, este profissional deverá buscar estratégias de enfrentamento para a prevenção de uma possível condição patológica. Com isso, é fundamental que o profissional reconheça, o seu nível de estresse individual e quais são os agentes estressores do ambiente de trabalho. Salienta-se ainda que a exposição continua dos profissionais de saúde aos fatores psicossociais, adversos ao meio ambiente, potencializam o risco de doenças cardiovasculares, musculoesqueléticas, gastrointestinais e psicológicas ${ }^{1,2}$.

A qualidade de vida no trabalho em unidades hospitalares, vem despertando o interesse de pesquisadores, tendo como evidência dois princípios observacionais, tais como: a carga horária de labuta excessiva e a rotina assistencial correlacionada com a dor, o sofrimento e a morte dos pacientes. Em paralelo, observa-se, que as unidades hospitalares aumentam a cobrança de produtividade dos profissionais de enfermagem, sem oferecer condições favoráveis para o desenvolvimento salutar e com prejuízos à saúde destes profissionais. Logo, a carga horária excessiva é um dos obstáculos preponderantes entre os profissionais da enfermagem, assim, podendo ocasionar acidentes de trabalho, devido ao cansaço mental e físico. Outro fator crucial que prevalece sobre o excesso de trabalho na saúde é o número insuficiente de profissionais de enfermagem, para realizar o atendimento assistencial aos pacientes. Ademais, o acúmulo de vínculos empregatícios tem provocado excesso de horas trabalhadas e, consequentemente, o tempo de lazer para as demais áreas da vida social é deixado em segundo plano. Em contrapartida, o mercado de trabalho tem se tornado rigoroso mediante o avanço tecnológico, exigindo que o profissional de saúde seja qualificado e especializado ${ }^{3,4}$.

Ademais, caracteriza-se o estresse como um conjunto de reações fisiológicas determinantes de situações inéditas e dependendo da intensidade e/ou duração do estresse desencadeia um desequilíbrio no organismo. Para tanto, conceitua-se que o estresse ocupacional é provenien- te do ambiente de trabalho, tendo como consequência, o resultado do colaborador frente ao estresse. Com isso, o estresse ocupacional apresenta fatores impares no ambiente laboral, tais como: organização, gestão, condiçôes estruturais e qualidade nas relações interpessoais no trabalho. Verificou-se que os principais fatores preponderantes do estresse ocupacional correspondem aos relacionamentos com colegas $(23,2 \%)$, as atividades atribuídas no trabalho $(62,8 \%)$ e o relacionamento com as chefias $(62,8 \%)^{1,2,3,4}$.

Destaca-se que os profissionais de enfermagem estão expostos a jornadas prolongadas, condições de trabalho inadequadas, utilização de equipamentos obsoletos, do ponto de vista ergonômico, permanecem muito tempo em pé, possuem poucas horas de sono e descanso. Desse modo, tais fatores são potencializadores do surgimento de lesões musculoesqueléticas e preditivos à exposição a riscos ocupacionais que refletem para o crescimento das taxas de afastamentos, licenças e aposentadorias por invalidez. Logo, a sobrecarga de atribuições contribui para que esses profissionais, apresentem semblantes exaustivos, refletindo na precariedade da assistência e a não formação de vínculos com o paciente. Logo, é natural que o corpo humano chegue ao limite físico, emocional e psíquico, desencadeado por fatores estressores laborais. Nesse sentido, faz-se premente que os gestores de 
saúde sejam participativos e demonstrem sensibilidade para identificar as necessidades de sua equipe ${ }^{5,6,7}$.

Outrossim, quando o trabalho de gestores das unidades hospitares é estruturado e organizado por uma equipe multidisciplinar e composto por trabalhadores de diferentes categorias profissionais, facilita e promove a visão holística e integral do estado de saúde do paciente. Para tanto, não é apenas a formação biomédica que devemos ter como essência exata, mas a estruturação de uma condição saudável dos profissionais de saúde, pautada no paciente e na unidade hospitalar. No tocante ao processo de cuidar, este sempre será o pilar para o atendimento das necessidades e prioridades individuais do paciente. Consequentemente, o modelo assitencial participativo, a não fragmentação do trabalho, a ampliação da efetividade do cuidado tornam o ambiente laboral acolhedor e dinâmico e distante dos fotores de estresse ocupacional ${ }^{8}$.

Como visto, o presente estudo busca fornecer elementos reflexivos acerca dos fatores que influenciam a qualidade de vida dos enfermeiros, bem como contribuir para o processo de humanização da assistência, visando proteger o trabalhador e promover melhores condições de vida dentro e fora das instituições de trabalho. Assim, objetivou-se identificar os fatores de estresse ocupacional e os comportamentos de saúde de enfermeiros de um hospital terciário de Fortaleza-CE.

\section{MÉTODO}

Trata-se de um estudo transversal, descritivo e de abordagem quantitativa, desenvolvido em uma unidade hospitalar de nível terciário de Fortaleza, cujo atendimento é exclusivo pelo Sistema Único de Saúde (SUS). A coleta de dados foi realizada no período de outubro de 2018 a março de 2019.

A amostra foi constituída por 134 enfermeiros que estão na prática assistencial, estimada a partir da fórmula de população finita. O cálculo amostral considerou uma prevalência média esperada de até $50 \%$
Como visto, o

presente estudo

busca fornecer

elementos reflexivos

acerca dos fatores

que influenciam a

qualidade de vida

dos enfermeiros,

bem como contribuir

para o processo

de humanização

da assistência,

visando proteger

o trabalhador e

promover melhores

condições de vida

dentro e fora das

instituições de

trabalho. para diversos indicadores, devido ao desconhecimento da proporção do evento, com nível de confiança de 95\% e com margem de erro de 5\%11. Para recrutamento dos participantes, adotou-se a amostragem por conveniência.

Os critérios de inclusão dos profissionais que participaram desse estudo foram: estar atuando diretamente na assistência e ter no mínimo seis meses de atuação profissional na instituição. Os critérios de exclusão consistiram em: licença médica, licença maternidade e férias no período de coleta de dados. Considerando-se os critérios de seleção e elegibilidade adotados para recrutamento dos participantes, os profissionais elegíveis foram devidamente localizados nos setores/unidades da instituição. Os participantes foram convidados a responder o questionário de pesquisa nas respectivas unidades/setores de atuação (ambulatórios, enfermarias e postos de enfermagem). Além disso, foi resguardado que o participante escolhesse o horário e o recinto das unidades/setores que lhes deixem mais à vontade para responder o instrumento.

$\mathrm{Na}$ coleta de dados, utilizou-se um instrumento, no formato de questionário estruturado, pré-codificado, com questões fechadas de múltipla escolha, com 12 perguntas relacionadas à percepção dos participantes acerca dos fatores de estresse ocupacional e comportamento de saúde. $\mathrm{O}$ instrumento de coleta de dados foi dimensionado em seis blocos, estruturados da seguinte forma: Bloco A - Caracterização da formação e profissional dos participantes da pesquisa; Bloco B - Situações de estresse relacionas ao ambiente laboral; Bloco C - Uso de substâncias psicoativas (tabaco, cafeína, medicamentos, álcool e outras drogas); Bloco D - Comportamentos de saúde adotados pelos profissionais de saúde; Bloco E - Sinais/sintomas de estresse profissional; Bloco F - Identificação dos principais fatores estressores dos profissionais participantes. O tempo previsto para aplicação do questionário foi de aproximadamente 15 minutos.

Os dados obtidos foram digitados e processados utilizando-se as planilhas 


\section{artigo}

Oliveira, F.A.; Melo, D.C.M.; Santos, A.P.L.M.; Menezes, C.P.S.R.; Sales, T.B.; Freitas, M.M.L.; Afonso, L.R.;

Estresse ocupacional e comportamentos de saúde de enfermeiros de um hospital terciário de Fortaleza - Ceará

Tabela 1. Características de formação e profissionais dos participantes (2019).

$$
\text { Variáveis: }
$$

\section{N}

Sexo

Mas

Fem

Idade

18 a 25 anos

26 a 33 anos

34 a 49 anos

50 a 65 anos

$>65$ anos

\section{Escolaridade}

Graduação

Especialização

Mestrado/Doutorado

Tempo de formado

$<1$ ano

1 a 5 anos

43

6 a 10 anos

11 a 15 anos

16 a 19 anos

$>20$ anos

Não respondeu

Tempo de serviço na instituição

$<1$ ano

1 a 5 anos

6 a 10 anos

11 a 19 anos

$>20$ anos

Não respondeu

Possui outro emprego

Sim

Não

Não respondeu

74

2

Vínculo trabalhista com a instituição

Cooperada(o)

Estatutária(o)

Celetista

Residente

Não respondeu

Fonte: Dados da pesquisa.
9 eletrônicas do programa Microsoft ${ }^{\oplus}$ Office Excel $^{\circ}$ 2016. Após tabulados, os dados foram analisados por estatística descritiva no programa STATA, calculando-se as frequências absolutas e relativas. A seguir, os resultados foram organizados em tabelas e analisados à luz da literatura científica pertinente ao tema.

Em atendimento aos aspectos éticos legais de pesquisa envolvendo seres humanos, o presente estudo recebeu autorização do serviço para sua realização e foi devidamente aprovado pelo Comitê de Ética e Pesquisa com seres humanos, sob parecer de $n^{\circ}$. 2.903.371.

\section{RESULTADOS}

Foram coletadas informações de 134 profissionais de enfermagem de hospital terciário de Fortaleza que atuam diretamente na assistência à saúde.

Observou-se que as mulheres são a principal força de trabalho do hospital, representando $91,0 \%(n=122)$ dos profissionais de saúde. Em relação à idade dos participantes, cerca de $60 \%(\mathrm{n}=79)$ têm idade entre 26 e 41 anos. Quanto à escolaridade, verificou-se que $15,7 \%(\mathrm{n}=21)$ possuem apenas a graduação, a grande maioria relatou possuir pós-graduação, sendo a especialização a mais frequente entre os participantes $(74,6 \%$; 100$)$, seguidos dos que possuem formação stricto sensu em nível de mestrado $(7,5 \% ; 10)$ e doutorado $(2,2 \% ; 3)$. Observou-se que $32,1 \%(\mathrm{n}=43)$ têm menos de 5 anos de formação e 23,9\% (n=32) têm entre 6 a 10 anos de formado. Contudo, identificou-se que cerca de um quarto dos participantes relataram possuir mais de 20 anos de formados $(24,6 \% ; 33)$.

Averiguou-se que cerca de $30 \%(\mathrm{n}=39)$ dos profissionais têm entre 6 a 10 anos de serviço na instituição, $25,4 \% \quad(n=34)$ têm de 1 a 5 anos e $21,6 \%(n=29)$ têm entre 6 e 12 meses e que aproximadamente $18,0 \%$ relataram ter mais de duas décadas de serviço. No que diz respeito ao vínculo de trabalho com a instituição, identificou-se que $32,1 \%(n=43)$ eram estatutários, os demais se encontravam na 
Tabela 2. Características relacionadas ao uso de substância psicoativas e comportamentos de saúde (2019).

\section{Variáveis:}

N

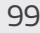

35

Sim

Tipo de substância*

Tabaco

Cafeína

Álcool

Medicamento

Comportamento de saúde

Não

$\operatorname{Sim}$

Tipo de comportamento de saúde*

Atividade física

63

47,0

Alimentação saudável

34

25,4

Tocar ou ouvir música

44

32,8

Lazer (ler livros, cinema/teatro/museu, praia, passeio, etc)

Uso de medicamento

Terapia

Atividades religiosas

4

Fonte: Dados da pesquisa. *Os participantes poderiam marcar mais de uma opção. condição de cooperados $(54,5 \% ; 73)$, celetistas $(10,5 \% ; 14)$ e residentes $(2,2 \% ; 3)$, respectivamente. Verificou-se que a maioria dos participantes só tem a instituição pesquisada como principal local trabalho (55,2\%; 74). Conforme mostra a Tabela 2, $26,1 \%(n=35)$ dos profissionais fazem uso de substâncias psicoativas. Dentre estas, a cafeína e os medicamentos são os dois mais frequentes, correspondendo a $17,9 \%$ $(n=24)$ e $11,2 \%(n=15)$, respectivamente.

Verificou-se que 47,0\% $(n=63)$ dos participantes realizavam ou praticavam algum comportamento de saúde. Dentre as atividades de comportamentos de saúde mais reportadas pelos profissionais, identificou-se 'lazer em geral' (ler livros, cinema/ teatro/museu, praia, passeio, etc) $(48,5 \%$; 65), prática de atividade física, tocar/ouvir música e ter alimentação saudável.

A seguir, a Tabela 3 reúne as características das variáveis relativas aos sintomas de estresse relatadas pelos enfermeiros. Verificou-se que $61,9 \%(n=83)$ revelaram apresentar de 1 a 5 sinais/sintomas de estresse, seguidos dos que relataram manifestar entre 6 a 10 sinais/sintomas $(26,1 \% ; 35)$.

Dentre os sinais/sintomas mais prevalentes de estresse, observou-se que 'tensão muscular', 'insônia' e 'desgaste físico” são sinais/sintomas reportados por $57,5 \%$ $(n=77), 55,2 \%(n=74)$ e $50,8 \%(n=68)$, respectivamente. Sinais/sintomas como 'angústia e/ou ansiedade e/ou medo diariamente', 'mudança de apetite' e 'agitação' também foram bem frequentes entre $30,6 \%$ a $36,6 \%$ dos profissionais de saúde.

Verificou-se que $56,7 \%(n=76)$ dos participantes apontam a 'Estrutura ambiental deficiente e falta de materiais' como principal fator de estresse, seguido de 'Insatisfação com a remuneração' e 'Ausência de reconhecimento profissional', ambos sinalizados por $53,0 \%(n=71)$.

O fator de estresse 'Processo de trabalho desgastante' foi elencado por cerca de $45,0 \%$ dos profissionais, acompanhado dos fatores 'Sobrecarga das atividades' e 'Carga horária excessiva”, ambos apontados por $39,6 \%(n=53)$ dos participantes do estudo. 


\section{artigo}

Oliveira, F.A.; Melo, D.C.M.; Santos, A.P.L.M.; Menezes, C.P.S.R.; Sales, T.B.; Freitas, M.M.L.; Afonso, L.R.;

Estresse ocupacional e comportamentos de saúde de enfermeiros de um hospital terciário de Fortaleza - Ceará

$\begin{array}{lcc}\text { Desgaste físico } & 68 & 50,8 \\ \text { Alterações dermatológicas } & 21 & 15,7 \\ \text { Alterações gástricas } & 30 & 22,4 \\ \text { Irritabilidade excessiva } & 38 & 28,4 \\ \text { Diminuição da libido } & 11 & 8,2 \\ \text { Náusea } & 15 & 11,2 \\ \text { Pesadelos frequentes } & 6 & 4,5 \\ \text { Apatia, depressão ou raiva prolongada } & 24 & 17,9 \\ \text { Cansaço constante e excessivo } & 49 & 36,6 \\ \text { Angustia e/ou ansiedade e/ou medo diariamente } & 33 & 24,6 \\ \text { Hipersensibilidade emotiva } & 22 & 16,4 \\ \text { Perda do senso de humor } & 24 & 17,9 \\ \text { Hipertensão } & 1 & 0,8 \\ \text { Ideação suicida } & 1 & 0,8 \\ \text { Fonte: Dados da pesquisa. } & & \end{array}$

Tabela 4. Identificação dos fatores de estresse relatados por enfermeiros (2019)

$$
\text { Variáveis: }
$$

Fator de estresse ocupacional

Não

Sim

Tipos de fatores de estresse

Estrutura ambiental deficiente e falta de materiais

Insatisfação com a remuneração

Sobrecarga das atividades

Dimensionamento de pessoal

Processo de trabalho desgastante

Trabalho noturno

Ausência de reconhecimento profissional

Acidente de trabalho

Relacionamento interpessoal com os demais profissionais

Trabalho repetitivo

Carga horária

Exposição constante a riscos

Lidar com a morte

Conflito entre o trabalho e a familia

Tomada de decisões de conflitos pessoais

Falta de autonomia profissional

Fonte: Dados da pesquisa.

\section{DISCUSSÃO}

O estudo revelou que as mulheres representam a principal força de trabalho da instituição, dentre os profissionais de saúde, dado que corrobora com o perfil da enfermagem no Brasil, o qual demonstrou que $85,1 \%$ dos profissionais são do sexo feminino. No que concerne à idade, verificou-se predominância na faixa etária entre 26 e 41 anos. Esses dados demonstram que a profissão de enfermagem é majoritariamente por mulheres, uma tendência histórica da profissão, embora, nas últimas décadas, se observe uma procura do curso por homens na área, correspondendo a $14,4 \%$ das vagas ${ }^{12,13}$.

Em relação à escolaridade, verificou-se que são poucos os profissionais que possuem apenas a graduação, tendo em vista que a grande maioria possui pós-graduação, sendo a especialização a mais frequente entre os participantes, o que demonstra que o mercado de trabalho tem requerido, cada vez mais, profissionais qualificados. Os estudos apontam que trabalhadores de saúde com especialização na área, podem facilitar a interação e integração com seu processo de trabalho, uma vez que há uma maior procura dos serviços de saúde por profissionais especializados. Além disso, recomenda-se que os profissionais, em especial os enfermeiros, busquem manter-se atualizados, adquirindo cada vez mais conhecimento técnico-científico, com vistas a favorecer as tomadas de decisões rápidas e assertivas, conduzindo segurança a toda equipe e, principalmente, visando a redução dos riscos que ameaçam a vida do paciente $e^{14,15}$.

No tocante ao tempo de formação e tempo de serviço na instituição, observou-se que a maioria dos participantes do estudo tem entre 6 a 10 anos de formado. Contudo, identificou-se que cerca de um quarto dos participantes relataram possuir mais de 20 anos de formados, dado representativo dos profissionais com mais de duas décadas de serviço de vínculo, os quais são os que apresentavam vínculo estatutário de trabalho. A literatura aponta que o tempo de serviço prolongado, faz com que os profissionais acabem se adaptando ao local, favorecendo menos indícios de estresse. Por outro lado, ter um grande tempo de serviço pode levar à banalização do seu processo de trabalho ${ }^{16}$.

Evidenciou-se que houve predomínio de profissionais com menor tempo de serviço na instituição entre os participantes, 
haja vista que a grande maioria relatou ter menos de uma década de trabalho no hospital. Esse dado equivale aos profissionais que prestam serviço nas modalidades cooperado, celetista e residente, respectivamente. Como consequências advindas do cenário de precarização do trabalho, apontam-se prejuízos para a saúde do trabalhador e para a organização devido à rotatividade de pessoal e à fuga de capital intelectual que interferem diretamente na qualidade da assistência ${ }^{17}$.

Observou-se, também, que havia um quinto de profissionais recém-formados, tendo entre 6 e 12 meses de instituição. Os resultados encontrados reforçam o processo de precarização da força de trabalho na enfermagem, decorrente da ausência de concursos públicos como forma de composição do quadro de pessoal permanente das instituições de saúde do país ${ }^{18}$.

Em relação ao tempo de atuação profissional, há autores que afirmam que os enfermeiros em início de carreira tendem apresentar maiores níveis de estresse quando se compara aos profissionais que já exercem a profissão a mais tempo. Nesse sentido, depreende-se que quanto maior o tempo de trabalho, menor o nível de estresse pelo fato de o enfermeiro ter adquirido maior segurança técnica e controle sobre as situações que surgem em sua rotina de trabalho ${ }^{19}$.

Constatou-se que a maioria dos participantes só tem a instituição pesquisada como principal local trabalho. Essa realidade deixa em evidência a dificuldade de se conciliar dois empregos, haja vista que, no contexto hospitalar onde a enfermagem apresenta-se como maior força de trabalho, as atividades são frequentemente complexas, além de estarem submetidos a uma rígida estrutura hierárquica para o cumprimento de horários, rotinas, normas e regulamentos ${ }^{20,21}$.

Identificou-se que os profissionais da saúde fazem uso de substâncias psicoativas e que dentre tais substâncias destacam-se: a cafeína e os medicamentos. Verificou-se que $37,4 \%$ dos profissionais faziam uso de medicamentos psicoativos, e alegavam que o uso está associado: às más condi-

\section{Dentre os sinais/}

sintomas mais

prevalentes de

estresse entre os

profissionais de

saúde, observou-se:

'tensão muscular',

'insônia', 'desgaste

físico', ‘angústia e/

ou ansiedade e/ou

medo diariamente',

'mudança de apetite'

e 'agitação'.

ções de trabalho, alta carga horária, várias noites de sono perdidas, tendo o estresse como principal motivo para o uso desses medicamentos. Constatou-se que o uso de substâncias psicoativas entre os profissionais enfermeiros é, em média, de 30 a 100 vezes mais prevalente nesses profissionais que em outros trabalhadores da saúde. Esses profissionais tornarem-se quimicamente dependentes, além de apresentarem um elevado grau de síndrome de exaustão, comparados com outros profissionais da saúde. Desse modo, a automedicação está mais suscetível aos profissionais de enfermagem, devido à maior possibilidade de autoadministração, a facilidade a essas substâncias em seu cotidiano de trabalho, haja vista que esses profissionais são res- ponsáveis ainda pelo o armazenamento $\mathrm{e}$ controle dessas substâncias ${ }^{22}$.

Verificou-se que a maioria dos profissionais pesquisados não realizavam a prática de algum comportamento e/ou atividade física, indicativo de um hábito/ estilo de vida saudável. Dentre as atividades de comportamentos de saúde mais reportadas pelos profissionais, identificou-se: 'lazer em geral', ler livros, cinema/ teatro/museu, praia, passeio, prática de atividade física, tocar/ouvir música e ter alimentação saudável. Vislumbra-se que a adoção de comportamentos saudáveis de saúde são atividades que ainda não fazem parte da realidade de hábitos regulares e saudáveis dos profissionais. Salienta-se que a adoção de comportamentos e estilo de vida saudáveis por profissionais de saúde é de fundamental importância para melhoria da qualidade de vida, tendo em vista o aumento das doenças crônicas não transmissíveis (DCNT's) na população geral, com destaque para a alimentação inadequada e o sedentarismo, decorrentes dos processos de industrialização, globalização e condições de trabalho ${ }^{23}$.

Acerca das características relativas aos sintomas de estresse relatados pelos enfermeiros, verificou-se que a maioria apresentou de 1 a 5 sinais/sintomas de estresse. Dentre os sinais/sintomas mais prevalentes de estresse entre os profissionais de saúde, observou-se: 'tensão muscular', 'insônia', 'desgaste físico', 'angústia e/ou ansiedade e/ou medo diariamente', 'mudança de apetite' e 'agitação'. Os sintomas do estresse comportam três fases: a primeira se refere à reação de defesa ou de alarme e que tem como sintomas a taquicardia, a palidez, a fadiga, a insônia e falta de apetite; a segunda fase é denominada como de resistência ou adaptativa, a qual o indivíduo evidencia sintomatologia relacionada ao isolamento social, à incapacidade de desligar-se do trabalho, à irritabilidade excessiva e à diminuição da libido; a terceira fase, por sua vez, se apresenta como estágio de exaustão ou esgotamento. Nesta fase, o indivíduo apresenta problemas como hipertensão arterial, depressão, ansiedade, problemas sexuais e dermatológicos, como 


\section{artigo}

Oliveira, F.A.; Melo, D.C.M.; Santos, A.P.L.M.; Menezes, C.P.S.R.; Sales, T.B.; Freitas, M.M.L.; Afonso, L.R.;

Estresse ocupacional e comportamentos de saúde de enfermeiros de um hospital terciário de Fortaleza - Ceará

psoríase, vitiligo, urticárias e alergia, além do infarto e até da morte súbita ${ }^{24}$.

Conforme exposto, observou-se que os profissionais relataram a presença de sintomas de estresse classificados nas três fases de ocorrência dos sintomas. Essa situação demonstra a realidade do processo de trabalho em que este está imbricado por situações estressoras, as quais estão ocasionando o surgimento de tais sintomas que podem implicar na saúde física e psicológica do profissional, assim, faz-se premente a implementação de estratégias e ações que mitiguem os fatores de estresse ocupacional.

Em relação à identificação de fatores estressores, observou-se que grande parte dos participantes aponta a 'Estrutura ambiental deficiente e falta de materiais' como principal fator de estresse, seguido de 'Insatisfação com a remuneração' e 'Ausência de reconhecimento profissional.' Também foram apontados como fatores de estresse ocupacional: 'Processo de trabalho desgastante,' 'Sobrecarga das atividades' e 'Carga horária excessiva?. Com isso, identificou-se que o estresse dos profissionais enfermeiros é favorecido pela: carga excessiva de trabalho, dificuldades relacionadas com o cliente e processos e estrutura organiza- cional da instituição hospitalar, portanto, caracteriza-se que a exaustão emocional, a despersonalização e a redução da realização pessoal correspondem às principais cargas estressoras ${ }^{25}$.

Por fim, observou-se que os principais fatores de estresse reportados pelos participantes deste estudo foram: o ambiente pessoal, a escala excessiva de trabalho, a falta de recursos para o desenvolvimento do processo de trabalho, a queda do desempenho da função, bem como das relações interpessoais entre colegas e/ou grupos no trabalho. Como visto, é necessário também que haja uma ação participativa e integral dos gestores, promovendo estratégias e intervenções que favoreçam melhores condiçôes de trabalho e, consequentemente, repercutam positivamente qualidade de vida dos profissionais.

\section{CONCLUSÃO}

A enfermagem é uma profissão vulnerável ao estresse em suas inumeráveis práticas diárias, sejam assistenciais ou gerenciais. Em virtude disso, buscou-se nesse trabalho identificar os fatores de estresse ocupacional e os comportamentos de saúde de enfermeiros de um hospital terciário de Fortaleza-CE. Acredita-se que o objetivo proposto foi alcançado, tendo em vista os resultados expressos acerca dos comportamentos de saúde dos profissionais, dos sintomas de estresses e dos seus respectivos fatores causadores, conforme se verificou ao longo do estudo.

Os resultados apresentados evidenciam a necessidade da adoção de medidas preventivas do estresse ocupacional no âmbito coletivo e organizacional, com vistas a proporcionar um ambiente de trabalho seguro e com recursos indispensáveis a uma assistência de qualidade. Compreende-se que a implementação de tais medidas pode contribuir para a promoção do bem-estar e a satisfação do grupo, resultando em melhoria do desempenho, minimizando, por conseguinte, os encargos sociais e financeiros para o indivíduo e a organização, considerando a possibilidade de redução dos riscos de adoecimento e absenteísmo.

Acredita-se que esse estudo será útil para que os gestores reflitam acerca dos fatores que influenciam a qualidade de vida dos enfermeiros e que estas reflexões possam contribuir para implementação de ações que melhorem as condições de trabalho, favorecendo a humanização da assistência e a proteção do trabalhador. •

\section{REFERÊNCIAS}

1. Ribeiro RP, Marziale MHP, Martins JT, Galdino MJQ, Ribeiro PHV. Estresse ocupacional entre trabalhadores de saúde de um hospital universitário. Rev. Gaúcha Enferm. [Internet]. 2018 [citado em 2020 Mai 31]; 39: e65127. Disponível em: $\quad$ http://www.scielo.br/scielo.php?script=sci_arttext\&pid=S1983-14472018000100421\&lng=en

2. Carvalho AEL, Frazão IS, Silva DMR, Andrade MS, Vasconcelos SC, Aquino JM. Estresse dos profissionais de enfermagem atuantes no atendimento pré-hospitalar. Rev. Bras. Enferm. [Internet]. 2020 [citado em 2020 Mai 31]; 73 (2): e20180660. Disponivel em: http://www.scielo.br/scielo.php?script=sci_arttext\&pid=S0034-71672020000200173\&lng=pt.

3. Dalri RCMB, Silva LA, Mendes AMOC, Robazzi MLCC. Carga horária de trabalho dos enfermeiros e sua relação com as reações fisiológicas do estresse. Rev. Latino-Am. Enfermagem [Internet]. 2014 Dez [citado em 2020 Jun 29]; 22 (6): 959-965. Disponivel em: http://www.scielo.br/scielo.php?script=sci_arttext\&pid=S0104-11692014000600959\&lng=en.
4. Araújo GA, Soares MJGO, Henriques MERM. Qualidade de vida: percepção de enfermeiros numa abordagem qualitativa. Rev. Eletr. Enfermagem [Internet]. 2009 [citado em 2019 fev. 02]; 11 (3): 635-641. Disponivel em: https://revistas.ufg.br/fen/article/ view/47180/23137

5. Santos EC, Andrade RD, Lopes SGR, Valgas C. Prevalência de dor musculoesquelética em profissionais de enfermagem que atuam na ortopedia. Rev. dor [Internet]. 2017 Dez [citado em 2020 Mai 31]; 18 (4): 298-306. Available from: http://www.scielo.br/scielo.php?script=sci_arttext\&pid=S1806-00132017000400298\&lng=en.

6. Zopi F, Fernandes P, Juliani C. Implicações da atividade do enfermeiro no dimensionamento da equipe de enfermagem na atenção primária à saúde. Rev Enfermagem UFPE on-line [Internet]. 2017 abr [citado em 29 jun 2020]; 11 (7): 2711-2717. Disponivel em: https://periodicos.ufpe.br/revistas/revistaenfermagem/article/view/23444/19148

7. Silveira TVL, Prado Júnior PP, Siman AG. Opinião dos enfermeiros sobre a utilização dos indicadores de qualidade na as- 


\section{REFERÊNCIAS}

sistência de enfermagem. Rev. Gaúcha Enferm. [Internet]. 2015 abr./jun. [citado em 2020 jun. 27]; 36 (2): 82-88. Disponivel em: https://seer.ufrgs.br/RevistaGauchadeEnfermagem/article/ view/47702

8. Merino-Plaza MJ, Carrera-Hueso FJ, Arribas-Boscá N Martínez-Asensi A, Vázquez-Ferreiro $P$, Vargas-Morales $A$ et al. Burnout y factores de riesgo psicosocial en el personal de un hospital de larga estancia. Cad. Saúde Pública [Internet]. 2018 [citado em 2020 Mai 31]; 34 (11): e 00189217. Disponivel em: http:/www.scielo.br/scielo.php?script=sci_arttext\&pid=S0102-311X2018001105011\&Ing=pt.

9. Hulley SB, Cummings SR, Browner WS, Grady DG, Deborah G, Newman TB et al. Delineando a pesquisa clínica: uma abordagem epidemiológica. $3^{a}$ ed. São Paulo: Artmed; 2008.

10. Dias NA, Hoepers NJ, Poluceno GAS, Ceretta LB, Schwalm MT, Soratto MT. Ocorrência de estresse entre acadêmicos de enfermagem de um curso noturno. Rev.Saúde.Com. [Internet]. 2014 [citado em 2020 jun 27]; 10 (21): 129-139. Disponivel em: http:// periodicos2.uesb.br/index.php/rsc/article/view/294

11. Machado $\mathrm{MH}$, Oliveira $\mathrm{E}$, Lemos W, Lacerda WF, Aguiar Filho W, Wermelinger $M$. et al. Mercado de trabalho da enfermagem: aspectos gerais. Enferm Foco [Internet]. 2016 [citado em 2020 jun. 27]; 7 (esp): 35-62. Disponivel em: http://revista.cofen.gov. br/index.php/enfermagem/article/view/691/301

12. Freitas RJM, Lima ECA, Vieira ES, Feitosa RMM, Oliveira GYM, Andrade LV. Estresse do enfermeiro no setor de urgência e emergência. Rev enferm UFPE on-line [Internet]. 2015 dez [citado em 2020 jun 27]; 9 (supl. 10): 1476-1483. Disponível em: https://periodicos.ufpe.br/revistas/revistaenfermagem/article/ view/10861

13. Urbanetto JS, Silva PC, Hoffmeister E, Negri BS, Costa BEP, Figueiredo CEP. Estresse no local de trabalho em trabalhadores de enfermagem de um hospital de emergência: análise da Job Stress Scale. Rev. Latino-Am. Enfermagem [Internet] 2011 Out [citado em 2020 jun 29]; 19 (5): 1122-1131. Disponivel em: http://www.scielo.br/scielo.php?script=sci_arttext\&pid=S0104-11692011000500009\&Ing=en.

14. Pereira CA, Miranda LCS, Passos JP. O Estresse ocupacional da equipe de enfermagem em setor fechado. J res: fundam care online [Internet]. 2009 set./dez. [citado em 2019 mar 10]; 1 (2): 196-202. Disponivel em: http://www.seer.unirio.br/index.php/ cuidadofundamental/article/view/346/331.

15. Oliveira EB, Barros PM, Perez Junior EF, Granadeiro DS, Xavier T, Rossone FO. Precarização do trabalho em serviço de emergência e dimensionamento de pessoal: um desafio para a gerência de enfermagem e a qualidade do serviço. In: Unicovsky MA, Waldman BF, Spezani RS, organizadores. Programa de atualização em enfermagem. Porto Alegre: Artmed Panamericana Editora; 2016.

16. França FM, Ferrari R. Síndrome de Burnout e os aspectos sócio-demográficos em profissionais de enfermagem. Acta paul. enferm. [Internet]. 2012 [citado em 2020 Jun 29]; 25 (5): 743-
748. Disponivel em: http://www.scielo.br/scielo.php?script=sci_arttext\&pid=S0103-21002012000500015\&lng=en.

17. Guido LA, Linch GFC, Pitthan LO, Umann J. Estresse, coping e estado de saúde entre enfermeiros hospitalares. Rev. esc. enferm. USP [Internet]. 2011 Dez [citado em 2020 Jun 29]; 45 (6): 14341439. Disponível em: http://www.scielo.br/scielo.php?script=sci_arttext\&pid=S0080-62342011000600022\&lng=en.

18. Marcitelli CRA. Qualidade de vida no trabalho dos profissionais de saúde. Ens. Ciên 2011; 15 (4): 215-228.

19. Maciel MPGS, Santana FL, Martins CMA, Costa WT, Fernandes LS, Lima JS. Uso de medicamentos psicoativos entre profissionais de saúde. Rev enferm UFPE on-line [Internet]. 2017 jul. [citado em 2020 jun 27]; 11 (Supl. 7): 2881-2887. Disponível em: https://periodicos.ufpe.br/revistas/revistaenfermagem/article/view/10177/19195

20. Rocha PR, David HMSL. Padrão de consumo de álcool e outras drogas entre profissionais de saúde: retrato de alunos de cursos lato sensu de uma instituição pública. SMAD Rev eletr saúde mental álc drog [Internet]. 2015 mar [citado em 2020 jun 27]; 11 (1): 42-48. Disponível em: http://pepsic.bvsalud.org/scielo. php?script=sci_arttext\&pid=S1806-69762015000100007\&Ing=pt\&nrm=iso\&tlng=pt

21. Neall RA, Atherton IM, Kyle RG. Nurses' health-related behaviours: protocol for a quantitative systematic review of prevalence of tobacco smoking, physical activity, alcohol consumption and dietary habits. J Adv Nurs. [Internet]. 2016 [citado em 2020 jun 27]; 72 (1): 197-204. Disponivel em: https://www. researchgate.net/publication/280583514_Nurses'_health-related_behaviours_Protocol_for_a_quantitative_systematic review_of_prevalence_of_tobacco_smoking_physical_activity_alcohol_consumption_and_dietary_habits

22. While AE. Are nurses fit for their public health role? Int I Nurs Stud. [Internet]. 2014 [citado em 2020 jun 27]; 51 (9): 11911194. Disponivel em: https://www.researchgate.net/publication/260151910_Are_nurses_fit_for_their_public_health_role

23. Azevedo VGB, Alves SM, Viana LVM, Veras KCBB, Carvalho $\mathrm{ML}$, Elias CMV. Estresse ocupacional em profissionais de enfermagem que atuam em unidades de urgência e emergência. Rev Interd. [Internet]. 2017 out./nov./dez. [citado em 2020 jun. 28] 10 (4): 112-124. Disponível em: https://revistainterdisciplinar. uninovafapi.edu.br/index.php/revinter/article/view/1339

24. Zétola VF, Pavanelli GM, Pereira GU, Germiniani FMB, Lange MC. Burnout syndrome: are stroke neurologists at a higher risk?. Arq. Neuro-Psiquiatr. [Internet]. 2019 Fev [citado 2020 Maio 31]; 77 (2): 84-90. Disponivel em: http://www.scielo.br/scielo.php?script=sci_arttext\&pid=S0004-282X2019000200084\&Ing=pt.

25. Silveira ALP, Colleta TCD, Ono HRB, Woitas LR, Soares SH, Andrade VLA et al. Síndrome de Burnout: consequências e implicações de uma realidade cada vez mais prevalente na vida dos profissionais de saúde. Rev Bras Med Trab. [Internet]. 2016 [citado em 2020 jun 28]; 14 (3): 275-284. Disponivel em: https:// cdn.publisher.gn1.link/rbmt.org.br/pdf/v14n3a13.pdf 